\title{
Radio Recombination Line Maser Objects: New Detections with the SMA
}

\author{
Izaskun Jiménez-Serra \\ Harvard-Smithsonian Center for Astrophysics, \\ 60 Garden Street, 02138 Cambridge, MA, USA \\ email: ijimenez-serra@cfa.harvard.edu
}

\begin{abstract}
Hydrogen radio recombination line (RRL) masers are a rare phenomenon in star forming regions. Since RRL masers were first detected in 1989 toward the emission line star MWC349A, several single-dish surveys at millimeter wavelengths have been carried out to detect other RRL maser objects. However, although RRL maser amplification is expected to appear at wavelengths $<2 \mathrm{~mm}$, MWC349A still remains as the only RRL maser object known to date. In this contribution, I will present our recent findings of two new RRL maser objects with the Submillimeter Array (SMA) toward the massive star forming regions Cepheus A HW2 and MonR2-IRS2. Sub-millimeter observations with interferometers such as the SMA and the Atacama Large Millimeter Array (ALMA) open the possibility to detect a much larger sample of RRL maser objects, where very detailed information about the kinematics and physical structure of the innermost ionized regions can be obtained toward these objects.
\end{abstract}

Keywords. masers, stars: formation, (ISM:) HII regions, techniques: interferometric

\section{Introduction}

Calculations of the level populations of atomic hydrogen in HII regions, very early showed that global population inversions could exist across the Rydberg levels of the hydrogen atom (Cillié 1936; Baker \& Menzel 1938). These population inversions lead to the formation of hydrogen recombination lines under non-LTE (Local Thermodynamic Equilibrium) conditions, so that stimulated emission can occur. This idea has been confirmed by detailed theoretical modeling of the non-LTE populations in recombining hydrogen (Walmsley 1990; Strelnitski, Ponomarev \& Smith 1996; Martín-Pintado 2002). In particular, this modeling shows that for ultracompact (UC) HII regions the measured electron densities $\left(N_{e} \sim 10^{4}-3 \times 10^{5} \mathrm{~cm}^{-3}\right.$; Wood \& Churchwell 1989) are high enough to significantly invert the level populations involved in RRL transitions at millimeter, submillimeter and far-IR wavelengths. Therefore, RRL maser amplification at wavelengths $<2 \mathrm{~mm}$ is expected to be a common phenomenon in massive star forming regions.

By carrying out single-dish observations of several RRLs toward the massive Young Stellar Object (YSO) MWC349A, Martín-Pintado, Bachiller \& Thum (1989) reported the first detection of strong RRL maser emission at $1.3 \mathrm{~mm}$ (i.e. for the $\mathrm{H} 29 \alpha$, H30 $\alpha$, and H31 $\alpha$ lines). The line profiles of the observed $1.3 \mathrm{~mm}$ RRLs are double-peaked, and their peak intensities are factors of $\geqslant 50$ brighter than the RRLs at $3 \mathrm{~mm}$. Since the derived line-to-continuum flux ratios toward MWC349A clearly exceed those predicted under LTE conditionst, Martín-Pintado, Bachiller \& Thum (1989) concluded that the RRLs detected at $1.3 \mathrm{~mm}$ toward MWC349A are masers.

$\dagger$ The line-to-continuum flux ratios provide a measurement of the departure from LTE for the observed RRLs, and are calculated as $\Delta v T_{L} / T_{C}$, with $\Delta v T_{L}$ the RRL integrated intensity and $T_{C}$ the free-free continuum flux at the frequency of the RRL, $\nu$. Under LTE and optically thin emission, $\Delta v T_{L} / T_{C} \propto \nu^{1.1}$ (Martín-Pintado, Bachiller \& Thum 1989). 
Since the discovery of RRL maser emission toward MWC349A, extensive searches of RRL masers have been carried out with single-dish telescopes toward other massive YSOs (Martín-Pintado 2002). However, MWC349A still remains as the only RRL maser object firmly reported to date. In this contribution, I will present the detection of two new RRL maser objects toward the Cepheus A HW2 and MonR2 star forming regions, carried out with the SMA.

\section{Extremely broad RRL maser emission toward Cepheus A HW2}

Cepheus A East is a very active region of massive star formation that shows a plethora of UC HII regions, water masers, and molecular outflows (e.g. Hughes \& Wouterloot 1984; Narayanan \& Walker 1996; Torrelles et al. 2011). The brightest radio continuum source, HW2, is a B0 star that has a rotating (and possibly photo-evaporating) molecular disk (Jiménez-Serra et al. 2007; Jiménez-Serra et al. 2009). In addition, HW2 powers a collimated, high-velocity ionized jet (Rodríguez et al. 1994), whose proper motions are consistent with expanding velocities $\geqslant 500 \mathrm{~km} \mathrm{~s}^{-1}$ (Curiel et al. 2006). This implies that if RRLs were detected toward this source, they would show extremely broad line profiles with linewidths $\geqslant 1000 \mathrm{~km} \mathrm{~s}^{-1}$.

Figure 1 reports the full spectra measured toward Cepheus A HW2 at the frequencies of the H40 $\alpha$, H34 $\alpha$, and H31 $\alpha$ RRLs. The observed spectra show three different features: i) a strong slope due to the increase of the dust continuum emission with frequency $\left(S_{\nu} \propto \nu^{\alpha}\right)$; ii) a forest of narrow molecular lines mostly arising from the HC source at 0.3 " east HW2 (Jiménez-Serra et al. 2009); and iii) three faint and extremely broad features extending in velocity from $\sim-500 \mathrm{~km} \mathrm{~s}^{-1}$ to $\sim 600 \mathrm{~km} \mathrm{~s}^{-1}$ (zero-intensity linewidths of $\sim 1100 \mathrm{~km} \mathrm{~s}^{-1}$ ). Jiménez-Serra et al. (2011) have proposed that these extremely broad features correspond to RRL emission arising from the high-velocity HW2 radio jet.

The RRLs detected toward Cepheus A HW2 show asymmetric double-peaked line profiles, with the blue-shifted component slightly brighter than the red-shifted gas (Figure 1). Like MWC349A, the derived line-to-continuum flux ratios for the RRLs at $2 \mathrm{~mm}$ and $1.3 \mathrm{~mm}$ are significantly larger than those predicted under LTE, indicating that the RRLs are likely affected by maser amplification (see Table 1 and Jiménez-Serra et al. 2011).

By using the 3D radiative transfer code of Martín-Pintado et al. (2011), we have modeled the intensity and distribution of the free-free continuum and RRL emission toward Cepheus A HW2. We have considered an isothermal $\left(T_{e} \sim 10^{4} \mathrm{~K}\right)$, collimated (semiopening angle $\sim 18^{\circ}$ ), and bi-conical jet, where the ionized gas is accelerated constantly to reach a velocity of $500 \mathrm{~km} \mathrm{~s}^{-1}$ at $35 \mathrm{AU}$ from the protostar. Our model not only reproduces the fluxes and morphology of the free-free continuum emission measured at centimeter wavelengths with the Very Large Array (VLA; Rodríguez et al. 1994), but also the extremely broad asymmetric profiles of the H40 $\alpha$, H34 $\alpha$, and H31 $\alpha$ RRLs.

Our model, however, fails to reproduce two features of the RRLs observed toward Cepheus A HW2: i) the peak intensities of the H34 $\alpha$ and $\mathrm{H} 31 \alpha$ lines, expected to be more affected by maser amplification; and ii) the velocity shift of $160 \mathrm{~km} \mathrm{~s}^{-1}$, with respect to the radial velocity of the source of $-10 \mathrm{~km} \mathrm{~s}^{-1}$, required to reproduce the lines. For the former feature, the discrepancies between the observed and predicted intensities are due to uncertainties in the LTE departure coefficients used in the model (see the contribution by A. Báez-Rubio in this volume). For the latter, inhomogeneities in the electron temperature, density and kinematics of the ionized gas in the inner regions of the jet could give rise to large asymmetries in the RRL profiles. From all this we suggest that the extremely broad features detected toward Cepheus A HW2 are associated with RRL masers generated in the high-velocity HW2 radio jet (Jiménez-Serra et al. 2011). 


\section{A new RRL maser object toward the MonR2-IRS2 source}

MonR2 is a well-known massive star forming cluster located at a distance of $950 \mathrm{pc}$. The brightest sources in the region are IRS1, IRS2 and IRS3. IRS1 $\left(L_{b o l} \sim 3 \times 10^{3} \mathrm{~L}_{\odot}\right.$; Henning, Chini \& Pfau 1992) is the exciting source of the evolved HII region detected with the VLA toward this region (Wood \& Churchwell 1989; Massi, Felli \& Simon 1985). IRS2 $\left(L_{b o l} \sim 6500 \mathrm{~L}_{\odot}\right.$; Henning, Chini \& Pfau 1992) is a very compact source responsible for the illumination of the inner walls of the HII region (Aspin \& Walther 1990). And IRS3 $\left(L_{b o l} \sim 1.4 \times 10^{4} \mathrm{~L}_{\odot}\right.$; Henning, Chini \& Pfau 1992) is a multiple source where the A and B objects show outflow activity (Preibisch et al. 2002). The MonR2 cluster is also associated with a large-scale CO outflow (total extent of 6.8 pc; Bally \& Lada 1983) seen in the northwest-southeast direction.

By using the SMA in the very extended (VEX) and compact configurations (COM), we have recently imaged the $\mathrm{H} 30 \alpha$ and H26 $\alpha$ RRL emission toward the MonR2 cluster. Figure 2 shows the VEX spectra (beam $~ 0.3 "-0.5 "$ ) of these lines extracted from the position of the MonR2-IRS2 continuum peak. The RRLs have double-peaked line

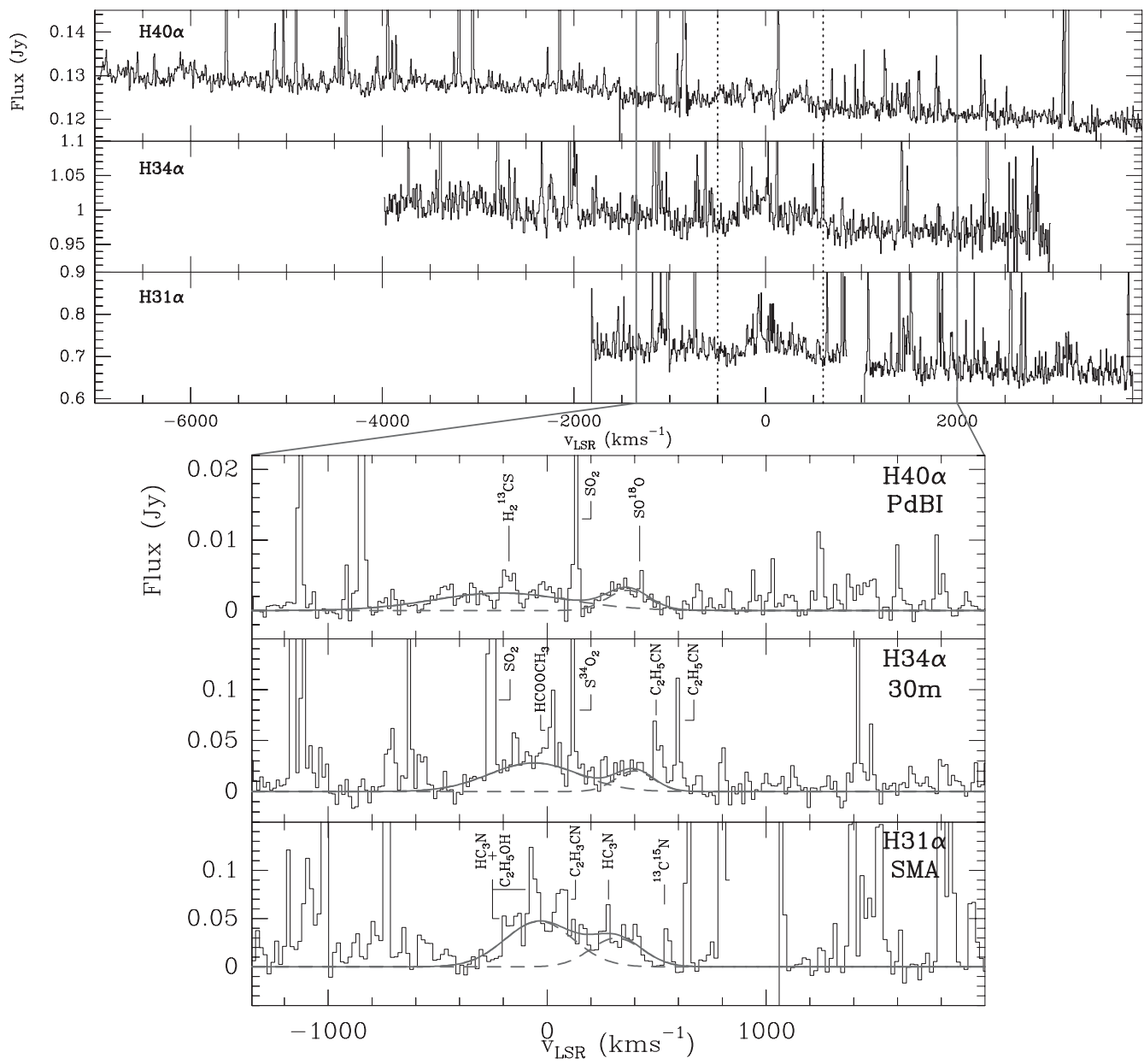

Figure 1. Upper panels: Full spectra of the $\mathrm{H} 40 \alpha, \mathrm{H} 34 \alpha$, and $\mathrm{H} 31 \alpha$ RRLs measured toward Cepheus A HW2 with the IRAM Plateau de Bure Interferometer (PdBI), the IRAM $30 \mathrm{~m}$ telescope, and the SMA. Lower panels: Zoom-in of the $\mathrm{H} 40 \alpha, \mathrm{H} 34 \alpha$, and $\mathrm{H} 31 \alpha$ spectra, showing the two-component Gaussian fits of the extremely broad RRLs (Jiménez-Serra et al. 2011). 
Table 1. Line-to-continuum flux ratios measured toward Cepheus A HW2 and MonR2-IRS2, and compared with those derived toward MWC349A.

\begin{tabular}{|c|c|c|c|c|c|c|c|}
\hline \multicolumn{5}{|c|}{ Cepheus A HW2 } & \multicolumn{3}{|c|}{ MonR2-IRS2 } \\
\hline RRL & $\begin{array}{c}\operatorname{LTE}^{a} \\
\left(\mathrm{~km} \mathrm{~s}^{-1}\right)\end{array}$ & $\begin{array}{c}\mathrm{HW}^{2} \\
\left(\mathrm{~km} \mathrm{~s}^{-1}\right)\end{array}$ & $\begin{array}{c}\text { MWC 349 } \\
\left(\mathrm{km} \mathrm{s}^{-1}\right)\end{array}$ & RRL & $\begin{array}{c}\mathrm{LTE}^{a} \\
\left(\mathrm{~km} \mathrm{~s}^{-1}\right)\end{array}$ & $\begin{array}{c}\text { IRS2 } \\
\left(\mathrm{km} \mathrm{s}^{-1}\right)\end{array}$ & $\begin{array}{c}\mathrm{MWC} 349 \\
\left(\mathrm{~km} \mathrm{~s}^{-1}\right)\end{array}$ \\
\hline $\mathrm{H} 40 \alpha$ & $\sim 30$ & 43 & $39^{b}$ & $\mathrm{H} 30 \alpha$ & $\sim 65$ & 90 & $298^{b}$ \\
\hline $\mathrm{H} 34 \alpha$ & $\sim 43$ & 229 & $110^{c}$ & $\mathrm{H} 26 \alpha$ & $\sim 103$ & 174 & $1660^{d}$ \\
\hline H31 $\alpha$ & $\sim 58$ & 280 & $215^{b}$ & $\ldots$ & $\ldots$ & $\ldots$ & $\ldots$ \\
\hline
\end{tabular}

Notes:

${ }^{a}$ Calculated for optically thin continuum emission, $T_{e}^{*}=10^{4} \mathrm{~K}$ and $N\left(\mathrm{He}^{+}\right) / N\left(\mathrm{H}^{+}\right)=0.08$.

${ }^{b}$ From Martín-Pintado, Bachiller \& Thum (1989).

${ }^{c}$ From Thum, Martín-Pintado \& Bachiller(1992).

${ }^{d}$ From Thum et al. (1994).

profiles resembling those measured toward MWC349A. The observed linewidths are $\sim 20$ $30 \mathrm{~km} \mathrm{~s}^{-1}$ and the RRL peaks are centered at velocities very different from that of the ambient cloud $\left(v_{L S R}=10 \mathrm{~km} \mathrm{~s}^{-1}\right.$; Figure 2$)$. While the H30 $\alpha$ red- and blue-shifted components have similar intensities, the intensity of the blue-shifted H26 $\alpha$ gas is a factor of $>3$ brighter than that of the red-shifted peak. This indicates that the RRL emission toward MonR2-IRS2 is affected by maser amplification. This is confirmed by the derived line-tocontinuum flux ratios (90 and $174 \mathrm{~km} \mathrm{~s}^{-1}$ for the H30 $\alpha$ and H26 $\alpha$ RRLs, respectively), which are clearly higher than those expected under LTE conditions (see Table 1).

The integrated intensity images of the red- and blue-shifted components of the $\mathrm{H} 26 \mathrm{\alpha}$ RRL toward MonR2-IRS2, reveal a small spatial shift (by $\leqslant 0.1$ ") in the east-west direction. This spatial shift could be interpreted as an ionized jet, like Cepheus A HW2, or as an ionized disk, as found toward MWC349A. Our SMA ${ }^{12} \mathrm{CO} J=2 \rightarrow 1$ COM data, however, do not show broad ${ }^{12} \mathrm{CO}$ emission arising from outflowing gas in the vicinity of IRS2. Therefore, the $\sim 0.1 "$ spatial shift between the red- and blue-shifted H26 $\alpha$ gas could be associated with a Keplerian rotating disk around a source with mass $\sim 9 \mathrm{M}_{\odot}$. This mass is consistent with the luminosity of the IRS2 source $\left(L_{b o l} \sim 6500 \mathrm{~L}_{\odot}\right)$. We propose that the RRL masers detected toward the YSO MonR2-IRS2 likely arise from an ionized circumstellar disk, in the same fashion as observed toward the massive YSO MWC349A.

We finally note that, in contrast with the VEX data, the H30 $\alpha$ RL data in COM configuration (beam $\sim 2.5 "-3.5$ ") show line emission with single-Gaussian profiles. This is probably due to the additional contribution arising from the large-scale and evolved IRS1 HII region within which the IRS2 source is embedded. This reveals that very highangular resolution observations are needed to pinpoint the regions where RRL maser

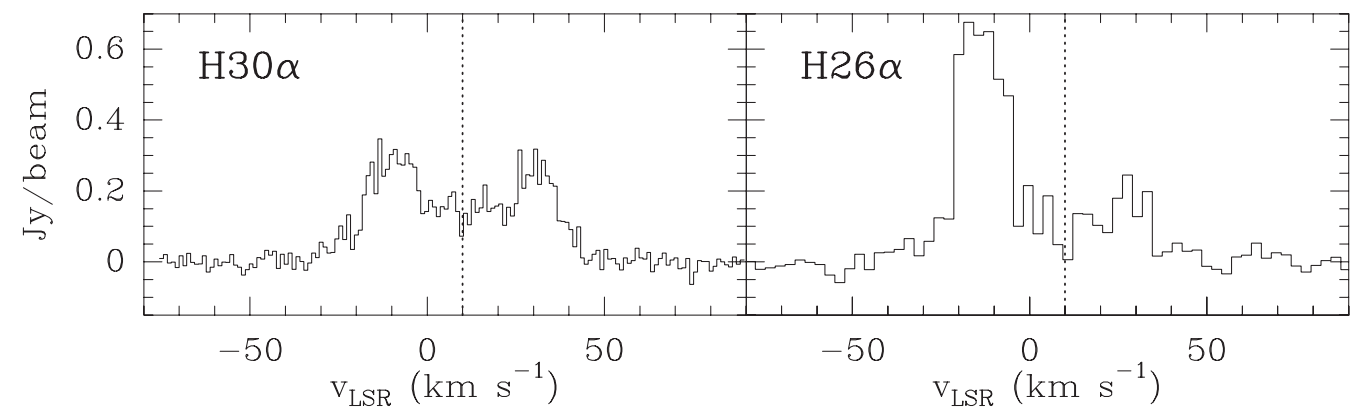

Figure 2. VEX spectra of the $H 30 \alpha$ and $H 26 \alpha$ RRLs extracted from the position of the MonR2-IRS2 continuum peak. The RRLs are double-peaked as in MWC349A. Vertical dotted lines show the central radial velocity of the MonR2 cloud $\left(v_{L S R}=10 \mathrm{~km} \mathrm{~s}^{-1}\right)$. 
amplification takes place, and it explains why single-dish surveys of RRL masers at $1.3 \mathrm{~mm}$ were unsuccessful in the past (Martín-Pintado 2002).

\section{Conclusions}

We report the detection of two new RRL maser objects toward the massive star forming regions Cepheus A HW2 and MonR2-IRS2. Our observations reveals that high-angular resolution interferometric observations at sub-millimeter wavelengths are strongly required to detect weakly amplified RRL masers such as those detected toward Cepheus A HW2 and MonR2-IRS2. Interferometric facilities such as the SMA and ALMA will be key in the detection of a larger sample of massive YSOs with RRL masers.

\section{References}

Aspin, C. \& Walther, D. M. 1990, A\&A, 235, 387

Baker, J. G. \& Menzel, D. H. 1938, ApJ, 88, 52

Bally, J. \& Lada, C. J. 1983, ApJ, 265, 824

Cillié, G. G. 1936, MNRAS, 96, 771

Curiel, S., et al. 2006, ApJ, 638, 878

Henning, Th., Chini, R., \& Pfau, W. 1992, A\&A, 263, 285

Hughes, V. A. \& Wouterloot, J. G. A. 1984, ApJ, 276, 204

Jiménez-Serra, I., Martín-Pintado, J., Rodríguez-Franco, A., Chandler, C., Comito, C., \& Schilke, P. 2007, ApJ, 661, L187

Jiménez-Serra, I., Martín-Pintado, J., Caselli, P., Martín, S., Rodríguez-Franco, A., Chandler, C., \& Winters, J. M. 2009, ApJ, 703, L157

Jiménez-Serra, I., Martín-Pintado, J., Báez-Rubio, A., Patel, N., \& Thum, C. 2011, ApJ, 732, L27

Martín-Pintado, J., Bachiller, R., \& Thum, C. 1989, A\&A 222, L9

Martín-Pintado, J. 2002, in: V. Mineese \& M. Reid K. K. (eds.) Cosmic Masers: From ProtoStars to Black Holes, IAU Symposium 206 (San Francisco: Astronomical Society of the Pacific), p. 226

Martín-Pintado, J., Thum, C., Planesas, P., \& Báez-Rubio, A. 2011, A\&̋A, 530, L15

Massi, M., Felli, M., \& Simon, M. 1985, A\& A, 152, 387

Narayanan, G. \& Walker, C. K. 1996, ApJ, 466, 844

Preibisch, T., Balega, Y. Y., Schertl, D., \& Weigelt, G. 2002, A\&A, 392, 945

Rodríguez, L. F., Garay, G., Curiel, S., Ramírez, S., Torrelles, J. M., Gómez, Y., \& Velázquez, A. 1994, ApJ, 430, L65

Strelnitski, V. S., Ponomarev, V. O., \& Smith, H. A. 1996, ApJ, 470, 1118

Thum, C., Martín-Pintado, J., \& Bachiller, R. 1992, A\&A, 256, 507

Thum, C., Matthews, H. E., Martín-Pintado, J., Serabyn, E., Planesas, P., \& Bachiller, R. 1994, $A \mathscr{E} A, 283,582$

Torrelles, J. M., et al. 2011, MNRAS, 410, 627

Walmsley, C. M. 1990, A\&ASS, 82, 201

Wood, D. O. S. \& Churchwell, E. 1989, ApJS, 69, 831 